\title{
Optimal relay allocation strategy based on maximum rate in two-user multi-relay system
}

\author{
Ji Liu $\cdot$ Xia Lei $\cdot$ Haibo Cao $\cdot$ Maozhu Jin
}

Received: 18 October 2012/Revised: 8 January 2013/Accepted: 15 January 2013/Published online: 5 June 2013

(C) The Author(s) 2013. This article is published with open access at Springerlink.com

\begin{abstract}
Cooperative communication can enhance the performance of wireless networks via relays, and so how to allocate the relay to obtain the optimal performance of system is a key issue. In this article, we consider a cooperative system where two users communicate with the destination via relays, and these relays connect with the destination by cable. Through the theoretical derivation and analysis, we obtain the optimal relay allocation strategy based on the maximum rate under the condition of relays setting forwarding thresholds. The result shows that the system has the maximum transmission rate when the relays are allocated equivalently between users. Moreover, compared with the single-user system, the results prove that diversity gain has a decisive effect on the performance in low SNR. However, with the SNR increasing, the impact of diversity gain on system rate will be reduced. In high SNR, spatial freedom degree of the channel of multiple users is brought to enhance the performance instead of diversity gain. Numerical results demonstrate the validity of the allocation strategy and conclusion arrived in this study, but we do not find the similar arguments in the literature so far, for comparison with the conclusion of this study.
\end{abstract}

Keywords Cooperative communication .

Relay allocation - Total rate

J. Liu $\cdot$ X. Lei $(\bowtie) \cdot$ H. Cao

National Key Laboratory of Communications,

University of Electronic Science and Technology of China,

Chengdu 610054, China

e-mail: leixia@uestc.edu.cn

M. Jin

Business School of Sichuan University, Chengdu 610065, China

\section{Introduction}

Recently, the cooperative communication has been explored more and more as an impressive technique to combat the effects of channel fading and to reduce the energy consumption in wireless networks [1,2]. In addition, cooperative communication efficiently reduces the probability of outage occurrence and solves the problems of intermittent communication and the signal detection in low signal-to-noise ratio (SNR) [3]. Moreover, the purposes of cooperative communication are to make full use of network nodes resources and to improve the transmission rate and reliability of wireless networks.

In cooperative systems, to utilize the wireless resources efficiently, relay allocations are often considered [4]. Bletsas et al. [5, 6] proposed an opportunistic relay which selects the relay with best channel condition cooperating source node for data transmission. The scheme does not require the use of complex space-time coding, but simultaneously reduce the resource consumption and simplify the information interaction between relays and destination. Hwang et al. [7] modified the basic opportunistic approach [5] by reducing the number of channel estimations. It defines a predefined SNR threshold, and the relay can take part in only if it satisfies such threshold. In [8] and [9], the sources include their residual power level on request-to-send (RTS) packets, allowing all overhearing nodes to estimate channel state information (CSI), thus making optimal power allocation. The relay selection decision depends upon the relay transmission power and CSI, as well as the residual power of source and relay nodes. In [10], the author developed a distributed relay selection for decode-and-forward (DF) cooperative networks, which uses an error-probabilityminimizing criterion to select relay and which can 
achieve full diversity without requiring the relay to know whether it decodes correctly. In the case of opportunistic relay selection in cooperative networks with secrecy constraints, where an eavesdropper node tries to overhear the source message, two new opportunistic relay selection techniques, which incorporate the quality of the relayeavesdropper links and take into account secrecy-related issues, were investigated by Krikidis [11]. Meanwhile, Zhang et al. [12] proposed an energy-efficient cooperative relay selection scheme that utilized the transmission power more efficiently in cooperative relay systems. Based on a suboptimal solution, the energy-efficient relay selection scheme was given, which selected the relay stations with the best energy efficiency and decided the optimal number of cooperative relay stations. In addition, threshold-based approaches which rely on a certain threshold to reduce the number of competing relays were addressed in [13] and [14].

The existing researches in the literature are based on the traditional cooperative model, relays of which primarily receive the information from the source and forward to the destination in the next stage [15-17]. Previous researches of relay allocations have obtained certain achievements. However, there are many studies [5-12] which require relays to know instantaneous CSI, and [13, 14] did not consider the performance of multiuser system. Compared with the traditional model, the cooperative model in which relays use the cable to receive signal instead of wireless medium has not been paid more attention, but still has important significance.

In this article, on the basis of the previous researches, we study the relay allocation strategy of two-user multi-relay system based on maximum rate by setting a forwarding threshold for relay without need to know the instantaneous CSI. With the proposed optimal relay allocation strategy, the performance of the two-user multi-relay system is compared with that of the single-user system, and the impacts that affect the rate of system are analyzed under different power transmission.

\section{System model}

In Fig. 1, we consider a wireless network composed of two users and $M$ relays, and the destination is a central node that receives the information from relays by cable. The relay nodes, which consist of large base stations, can communicate with each other through the central node in order to get the state information of the other relays. In this article, we mainly solve the problem of the relay allocation between users.

Orthogonal transmissions are used for simultaneous transmissions among different users by using different

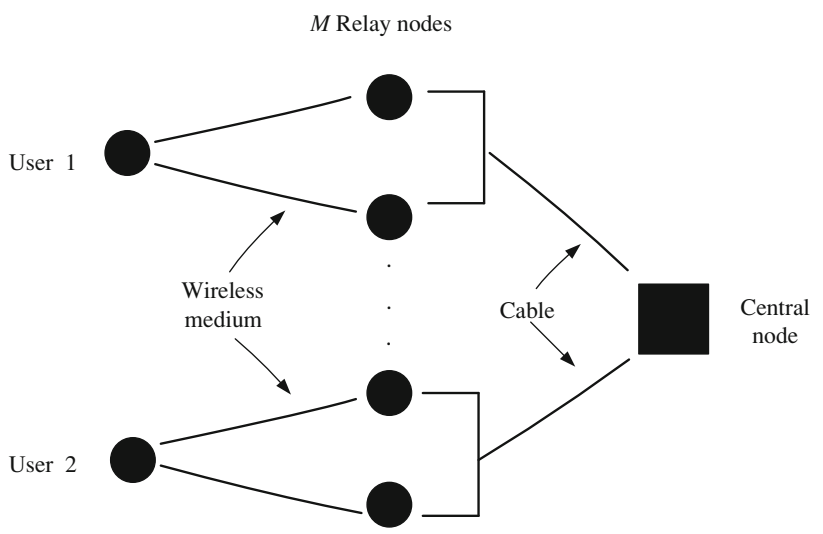

Fig. 1 The diagram of two-user $M$-relay system

channels (e.g., with different frequency bands) and time division multiplexing is employed for each user [18]. Let $h_{i, j}$, where $i=1,2$ and $j=1,2, \ldots, M$, denote the channel between the $i$ th user and the $j$ th relay node. $\left|h_{i, j}\right|$ obeys Rayleigh distribution.

$R_{i}$, the transmission rate of the $i$ th user, is defined as [19]

$R_{i}=\log _{2}\left(1+\sum_{j \in S_{i}}\left|h_{i, j}\right|^{2} \cdot r_{0}\right)$,

where $r_{0}$ represents the SNR with the noise environment alone, and $\boldsymbol{S}_{i}$ is defined as the set of relays allocated to $i$ th user. The maximum-ratio-combining (MRC) is employed at each relay set, and the combined signals are transmitted to the central node by cable.

\section{The relay allocation strategy of two-user $M$-relay system}

In this section, we consider the single-user and the two-user systems.

According to (1), $R_{1-M}$ is given by

$$
R_{1-M}=\log _{2}\left(1+\sum_{j=1}^{M}\left|h_{j}\right|^{2} \cdot r_{0}\right)=\log _{2}(1+r),
$$

where $r$ represents the receiving SNR of the combined signals from relay sets. If the channel power is normalized, then $\left|h_{j}\right|^{2}$ obeys an exponential distribution with $\lambda=1$.

The probability density function (PDF) of the sum of $N$ random variables with the same exponential distribution is given by [20]

$f_{X_{1}+X_{2}+\cdots+X_{n}}(x)=\frac{\lambda^{n}}{(n-1) !} x^{n-1} \exp (-\lambda x)$. 
The PDF of $r$ is written as

$f_{r}(x)=\frac{1}{r_{0} \cdot(M-1) !}\left(\frac{x}{r_{0}}\right)^{M-1} \exp \left(-\frac{x}{r_{0}}\right)$.

The distribution function of $R_{1-M}$ is given by

$F_{R_{1-M}}(y)=P(Y<y)=P\left(\log _{2}(1+r)<y\right)=\int_{0}^{2^{y}-1} f_{r}(x) \mathrm{d} x$.

The PDF of $R_{1-M}$ is given by

$$
\begin{aligned}
f_{R_{1-M}}(y)= & F_{R_{1-M}}^{\prime}(y)=\frac{2^{y} \cdot \ln 2}{r_{0} \cdot(M-1) !}\left(\frac{2^{y}-1}{r_{0}}\right)^{M-1} \\
& \cdot \exp \left(-\frac{2^{y}-1}{r_{0}}\right) .
\end{aligned}
$$

The rate of single-user $M$-relay system is

$$
R_{1-M}=E_{1-M}(Y)=\int_{0}^{\infty} y \cdot f_{R_{1-M}}(y) \mathrm{d} y \text {. }
$$

In addition, for the two-user $M$-relay system, assume that the power of transmission is normalized. Referring to (1), $R_{2-M}$ is given as follows:

$$
\begin{aligned}
R_{2-M} & =\log _{2}\left(1+\sum_{i \in S_{1}}\left|h_{i}\right|^{2} \cdot \frac{1}{2} r_{0}\right)+\log _{2}\left(1+\sum_{j \in S_{2}}\left|h_{j}\right|^{2} \cdot \frac{1}{2} r_{0}\right) \\
& =\log _{2}\left(1+r_{1}\right)+\log _{2}\left(1+r_{2}\right)=R_{1}+R_{2}
\end{aligned}
$$

where user 1 is allocated $\left|\boldsymbol{S}_{1}\right|=a$ relays and user 2 is allocated $\left|\boldsymbol{S}_{2}\right|=M-a$ relays. Assuming that the channels between each user and relays are independent, $r_{1}$ and $r_{2}$ are independent, identical distributed.

In a similar way, according to (6), the PDFs of $R_{1}$ and $R_{2}$ are expressed as follows:

$$
\begin{aligned}
f_{R_{1}}(y)= & \frac{2^{y} 2^{a} \ln 2}{r_{0}(a-1) !} \cdot\left(\frac{2^{y}-1}{r_{0}}\right)^{a-1} \times \exp \left(-\frac{2\left(2^{y}-1\right)}{r_{0}}\right) \\
f_{R_{2}}(y)= & \frac{2^{y} 2^{M-a} \ln 2}{r_{0}(M-a-1) !} \cdot\left(\frac{2^{y}-1}{r_{0}}\right)^{M-a-1} \\
& \times \exp \left(-\frac{2\left(2^{y}-1\right)}{r_{0}}\right) .
\end{aligned}
$$

Then, the PDF of $R_{2-M}=R_{1}+R_{2}$ is given by [20]

$$
\begin{aligned}
f_{R_{2-M}}(y) & =\int_{-\infty}^{+\infty} f_{R_{1}}(\tau) \cdot f_{R_{2}}(y-\tau) \mathrm{d} \tau \\
& =\int_{0}^{y} f_{R_{1}}(\tau) \cdot f_{R_{2}}(y-\tau) \mathrm{d} \tau .
\end{aligned}
$$

Thus, (8) is also expressed as

$$
\begin{aligned}
R_{2-M} & =E_{2-M}(Y)=\int_{0}^{\infty} y \cdot f_{R_{2-M}}(y) \mathrm{d} y \\
& =\int_{0}^{\infty} y \cdot \int_{0}^{y} \frac{2^{M} 2^{y}(\ln 2)^{2}}{r_{0}^{2}(a-1) !(M-a-1) !} \times\left(\frac{2^{\tau}-1}{r_{0}}\right)^{a-1} \\
& \times\left(\frac{2^{y-\tau}-1}{r_{0}}\right)^{M-a-1} \times \exp \left(-\frac{2\left(2^{\tau}+2^{y-\tau}-2\right)}{r_{0}}\right) \mathrm{d} \tau \mathrm{d} y .
\end{aligned}
$$

Using numerical integration in (12), we can obtain the rates $R_{2-M}$ of different relay allocation strategies in definite $r_{0}$. Furthermore, considering $M$ as even number, we obtain the maximum rate $R_{2-M}^{\max }$ at $a=M / 2$.

When we compare the single-user system with the twouser system, (2) and (8) will be analyzed. Let

$A=\sum_{j=1}^{M}\left|h_{j}\right|^{2}, \quad B=\sum_{i \in S_{1}}\left|h_{i}\right|^{2} \quad$ and $\quad C=\sum_{j \in S_{2}}\left|h_{j}\right|^{2}$,

and let us assume that the power of each channel is normalized; then we get $A=B+C$. If the relays are allocated equivalently between users, then $B=C=A / 2$.

Let

$1+A r_{0}=\left(1+\frac{1}{2} B r_{0}\right)\left(1+\frac{1}{2} C r_{0}\right)$.

We get a cross point $r_{0}^{\prime}=\frac{2 A}{B C}$. Then, if the number of relays allocated between users is equal, $r_{0}^{\prime}=\frac{8}{A}$. Therefore, if SNR $r_{0}<r_{0}^{\prime}$, the rate of single-user system is higher; on the contrary, it is the two-user system.

\section{The relay allocation strategy of relay node with a forwarding threshold}

When relays set the forwarding thresholds, it means that if the SNR of the combined signal is greater than $r$ th, the relays will receive the information and forward to the central node; otherwise, the information will be discarded. In this way, the receiving complexity of system can be reduced.

Similar to the above analysis, for the single-user $M$-relay system, after relays set the forwarding thresholds, the PDF of $R$ is expressed as

$f_{R}^{\text {th }}(y)=\left\{\begin{array}{cc}\int_{0}^{\log _{2}\left(1+r_{\text {th }}\right)} f_{R}(x) \mathrm{d} x, & y=0, \\ f_{R}(y), & y>\log _{2}\left(1+r_{\text {th }}\right) .\end{array}\right.$

Then, the rate of the single-user system is given by 


$$
R_{1-M}^{\mathrm{th}}=E_{1-M}^{\mathrm{th}}(Y)=\int_{\log _{2}\left(1+r_{\mathrm{th}}\right)}^{\infty} y f_{R}^{\mathrm{th}}(y) \mathrm{d} y .
$$

For the two-user $M$-relay system, referring to (9) and (10), after relays set the forwarding thresholds, let $f_{R_{1}}^{\text {th }}(y)$ denote the PDF of rate at user 1 to whom $a$ relays are allocated, and $f_{R_{2}}^{\text {th }}(y)$ denote the PDF of rate at user 2 to whom $M-a$ relays are allocated. Then, the PDF of the total rate $R$ is written as

$$
\begin{aligned}
f_{R_{2-M}}^{\mathrm{th}}(y) & =f_{R_{1}}^{\mathrm{th}}(y) * f_{R_{2}}^{\mathrm{th}}(y) \\
& = \begin{cases}a \cdot b, & y=0, \\
\int_{\log _{2}\left(1+\log _{2}\left(1+r_{\text {th }}\right)\right.}^{y} f_{R_{1}}(\tau) f_{R_{2}}(y-\tau) \mathrm{d} \tau \\
+a \cdot f_{R_{1}}(y)+b \cdot f_{R_{2}}(y), & y>\log _{2}\left(1+r_{\mathrm{th}}\right),\end{cases}
\end{aligned}
$$

where

$$
\begin{aligned}
& a=\int_{0}^{\log _{2}\left(1+r_{\mathrm{th}}\right)} f_{R_{2}}(x) \mathrm{d} x, \quad \text { and } \\
& b=\int_{0}^{\log _{2}\left(1+r_{\mathrm{th}}\right)} f_{R_{1}}(x) \mathrm{d} x .
\end{aligned}
$$

Therefore, the total transmission rate of the system is given as follows:

$R^{\text {th }}=E(Y)=\int_{\log _{2}\left(1+r_{\mathrm{th}}\right)}^{\infty} y \cdot f_{R_{2-M}}^{\text {th }}(y) \mathrm{d} y$.

In a similar way to Sect. 3, after relays set the forwarding thresholds, we also reach the cross point $r_{0 \text { th }}^{\prime}=\frac{2 A}{B C}$. Owing to the relays discard, the SNR signals of which are less than the threshold, the receiving SNR will decrease for the equal transmission power in low SNR. Thus, the user needs more power to reach the same transmission rate, $r_{0 \text { th }}^{\prime}>r_{0}^{\prime}$. With the increase of threshold $r_{\mathrm{th}}, r_{0 \text { th }}^{\prime}$ will be larger. Likewise, with the increasing number of relays, diversity gain will be enhanced. In the case when other conditions remain unchanged, the required power to reach the same transmission rate will be lower, and $r_{0 \text { th }}^{\prime}$ will decrease.

\section{Simulation results}

In this section, simulations are performed to demonstrate the above theory. In the Rayleigh channel, by setting different thresholds for relays, we compared different relay allocation strategies between theory and simulation. The simulation parameters are shown in Table 1.
Table 1 Simulation parameters

\begin{tabular}{ll}
\hline Channel & Rayleigh \\
\hline Number of users & 1,2 \\
Number of relay nodes & 4,6 \\
Forwarding threshold of relay & $0,8,10 \mathrm{~dB}$ \\
Transmitted total power & Normalization
\end{tabular}

Figures 2 and 3 illustrate the total transmission rates of different relay allocation strategies without the forwarding threshold of relay. For the two-user $M$-relay system, the allocation scheme in which the relays are allocated equivalently between users can get the maximum rate. Comparing the two-user system with the single-user system, we can find the cross point SNR $r_{0}^{\prime}$ in the figures. If

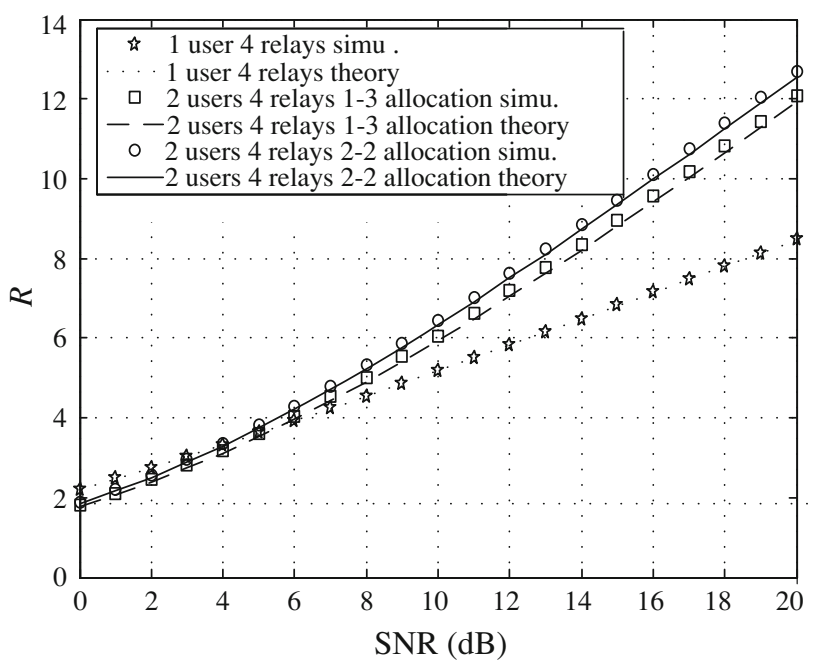

Fig. 2 Total transmission rates of 4-relay systems without threshold

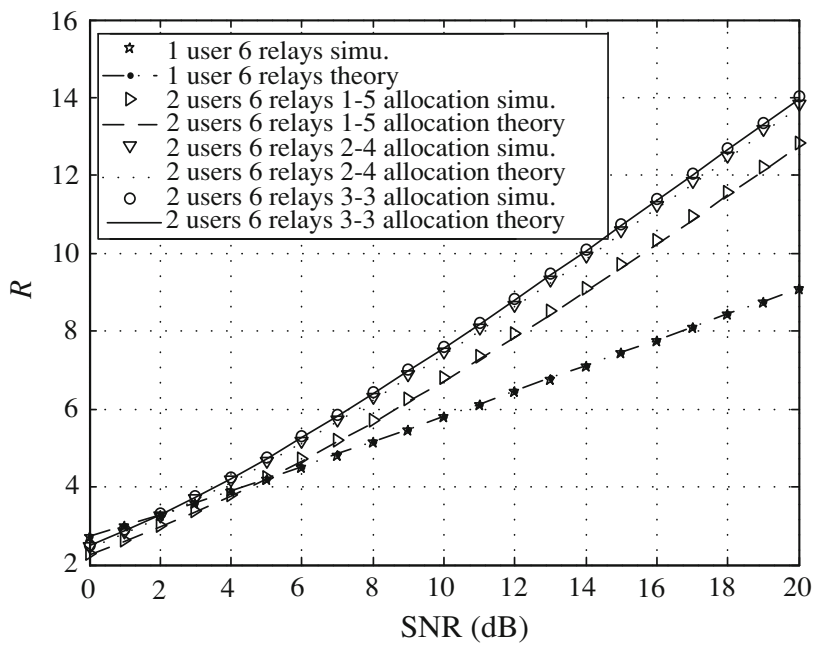

Fig. 3 Total transmission rates of 6-relay systems without threshold 
SNR $r_{0}<r_{0}^{\prime}$, the rate of the single-user system is higher; otherwise, the two-user system has a higher rate. In addition, with the number of relays increasing, the system can get more diversity gain. This means that the required power to reach the same rate will be lower, and $r_{0 \text { th }}^{\prime}$ will be greater.

Figures 4 and 5 illustrate the total transmission rates of different relay allocation strategies with $8 \mathrm{~dB}$ forwarding thresholds of relays. Compared with Figs. 2 and 3, the combined signals less than the threshold are discarded. The total rate of system is decreased in low SNR, and the required power to reach the same rate will be increased in

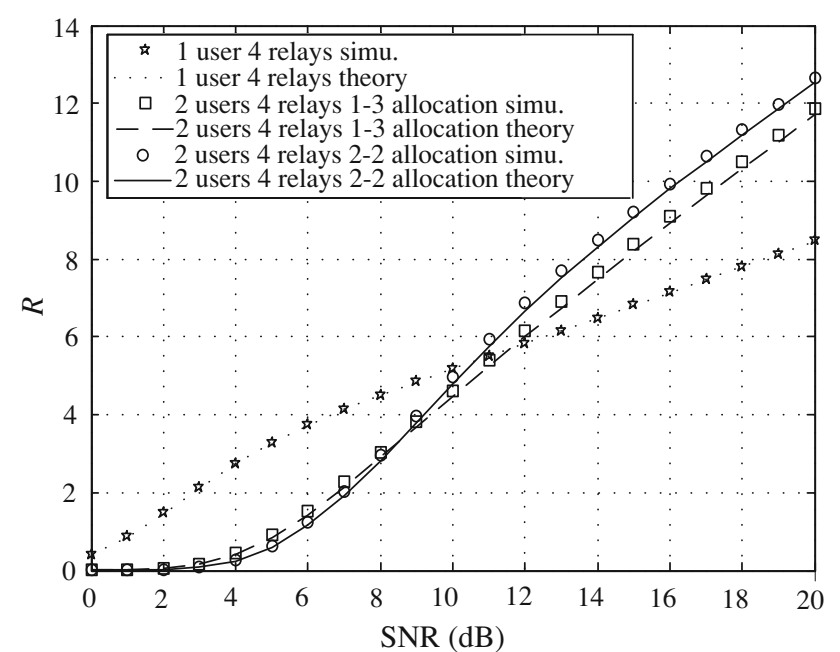

Fig. 4 Total transmission rates of 4-relay systems with $8 \mathrm{~dB}$ threshold

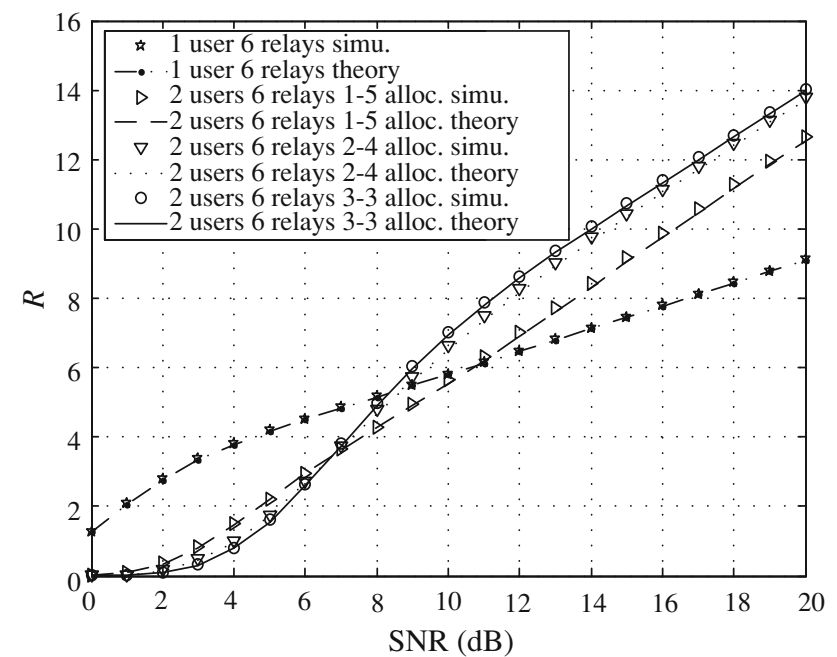

Fig. 5 Total transmission rates of 6-relay systems with $8 \mathrm{~dB}$ threshold contrast to the threshold-free case. Therefore, when the threshold is set, the cross point $r_{0 \text { th }}^{\prime}$ will be increased.

Figures 6 and 7 illustrate the total transmission rates of different relay allocation strategies with increasing forwarding threshold. Compared with Figs. 4 and 5, the number of the discarded signals is enlarged. Under the unchanged channel condition, the acquired power to reach the same rate will increase in low SNR. Therefore, with the thresholds increasing, the cross point $r_{0 \text { th }}^{\prime}$ will increase.

By analyzing the single-user and the two-user systems with the same number of relays in the simulations, we found that diversity gain decided the system performance

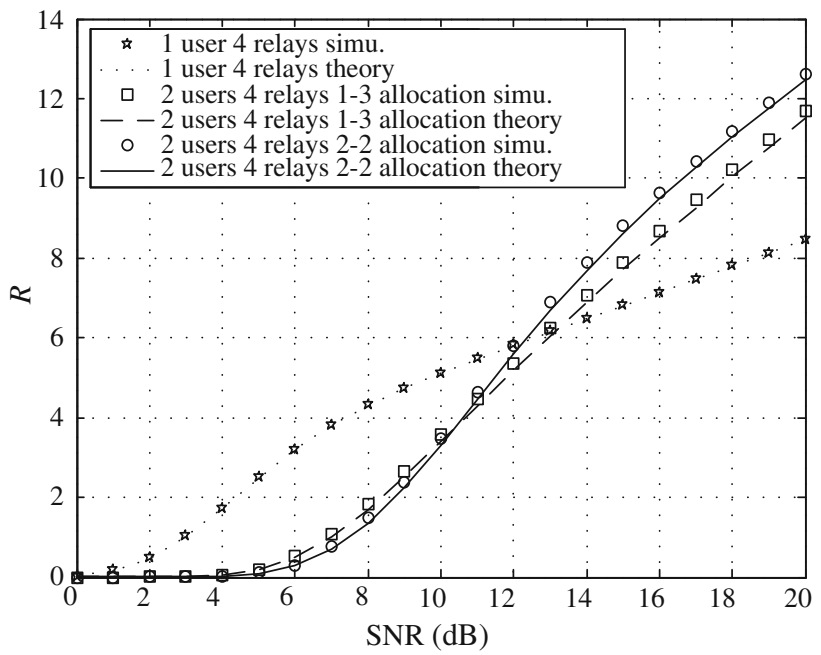

Fig. 6 Total transmission rates of 4-relay systems with $10 \mathrm{~dB}$ threshold

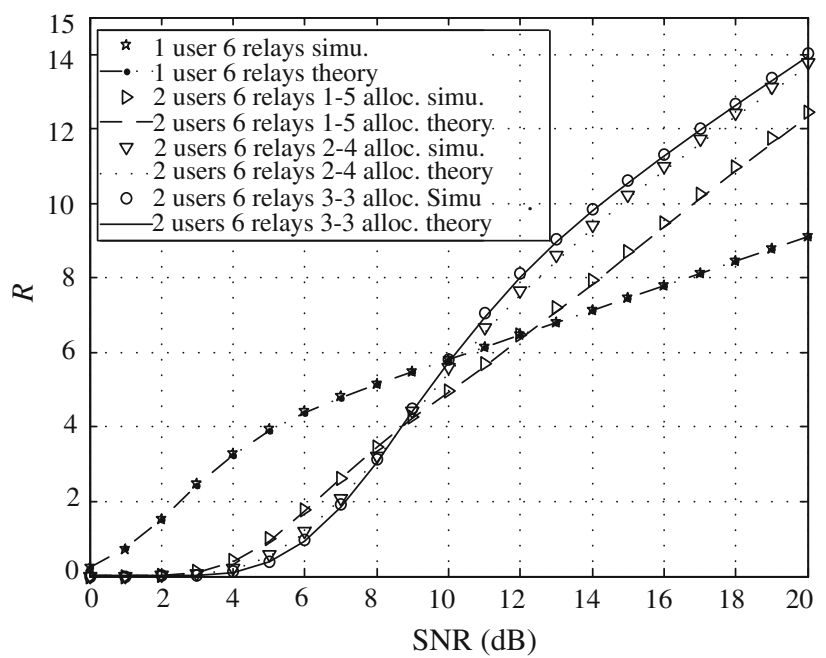

Fig. 7 Total transmission rates of 6-relay systems with $10 \mathrm{~dB}$ threshold 
in low SNR. Moreover, the transmission rate of the singleuser system is higher than that of the two-user system, because the diversity gain of the single-user system is larger. In high SNR, the impact of diversity gain on system rate decreased. With the SNR increasing, the impact can be ignored, and the spatial freedom degree of the channel is brought to enhance the performance in multiple users instead of diversity gain.

\section{Conclusion}

This article mainly investigates how to allocate the relays to obtain the optimal performance of system. Under the conditions of relays setting forwarding thresholds, a twouser multi-relay system was analyzed. The results show that the system can obtain the maximum transmission rate when the relays are allocated equivalently between users. In addition, comparison between the two-user system with the single-user system proves that diversity gain has a decisive effect on the performance in low SNR. Moreover, the single-user system can obtain the maximum transmission rate because of the larger diversity gain. However, with the SNR increasing to a certain value, the impact of diversity gain on system rate will reduce. In high SNR, the spatial freedom degree of the channel of multiple users instead of the diversity gain will improve the performance effectively.

Acknowledgments This study was supported by the National Natural Science Foundation of China under Grant numbers 61032002, 61101090, and 60902026, and the Chinese Important National Science \& Technology Specific Projects under Grant number 2011ZX03001-007-01.

Open Access This article is distributed under the terms of the Creative Commons Attribution License which permits any use, distribution, and reproduction in any medium, provided the original author(s) and the source are credited.

\section{References}

1. Laneman JN, Tse DNC, Wornell GW (2004) Cooperative diversity in wireless networks: efficient protocols and outage behavior. IEEE Trans Inform Theory 50(12):3062-3080

2. Jamal T, Mendes P (2010) Relay selection approaches for wireless cooperative networks, In: 2010 IEEE 6th international conference on wireless and mobile computing, networking and communications, Niagara Falls, Ontario, 11-13 Oct 2010, pp 661-668
3. Morillo-Pazo J, Trullols, Barcelo JM et al (2008) A cooperative ARQ for delay-tolerant vehicular networks, In: Proceedings of IEEE ICDCS, Beijing, June 2008, pp 192-197

4. Wei Y, Yu FR, Song M (2010) Distributed optimal relay selection in wireless cooperative networks with finite-state Markov channels. IEEE Trans Veh Technol 59(5):2149-2158

5. Bletsas A, Khisti A, Reed D et al (2006) A simple cooperative diversity method based on network path selection. IEEE J Sel Areas Commun 24(3):659-672

6. Bletsas A, Lippnian A, Reed DP (2005) A simple distributed method for relay selection in cooperative diversity wireless networks, based on reciprocity and channel measurements, In: Proceeding of IEEE 61st vehicular technology conference, Stockholm, May 30-June 1 2005, pp 1484-1488

7. Hwang KS, Ko YC (2007) An efficient relay selection algorithm for cooperative networks, In: Proceedings of IEEE VTC, Baltimore, Sept 30-Oct 3 2007, pp 81-85

8. Chen Y, Yu G, Qiu P et al (2006) Power-aware cooperative relay selection strategies in wireless ad hoc networks, In: Proceedings of IEEE PIMRC, Helsinki, Sep 2006, pp 1-5

9. Adam H, Bettstetter C, Senouci SM (2008) Adaptive relay selection in cooperative wireless networks, In: Proceedings of IEEE PIMRC, Cannes, Sept 2008, pp 1-5

10. Liu J, Lu K, Cai X (2010) Distributed error-probability-minimizing relay selection for cooperative wireless networks, In: 2010 44th annual conference on information sciences and systems, Princeton, 17-19 Mar 2010, pp 1-6

11. Krikidis I (2010) Opportunistic relay selection for cooperative networks with secrecy constraints. Commun IET 4(15):1787-1797

12. Zhang T, Zhao S, Cuthbert L et al (2010) Energy-efficient cooperative relay selection scheme in MIMO relay cellular networks. In: IEEE international conference on communication systems (ICCS), Singapore, 17-19 Nov 2010, pp 269-273

13. Huang KS, Ko YC (2007) An efficient relay selection algorithm for cooperative networks. In: Proceedings of IEEE VTC, Baltimore, Sept 30-Oct 3 2007, pp 81-85

14. Bali Z, Ajib W, Boujemaa H (2010) Distributed relay selection strategy based on source-relay channel. In: 2010 IEEE 17th international conference on telecommunications, Doha, 4-7 Apr 2010, pp 138-142

15. Zhang J, Zhuang H, Li T et al (2009) A novel relay selection strategy for multi-user cooperative relaying networks. In: IEEE 69th vehicular technology conference, Barcelona, 26-29 Apr 2009, pp 1-5

16. Hosseini K, Adve R (2010) Comprehensive node selection and power allocation in multi-source cooperative mesh network. In: 2010 44th annual conference on information sciences and systems, Princeton, 17-19 Mar 2010, pp 1-6

17. Baidas MW, Mackenzie AB (2012) An auction mechanism for power allocation in multi-source multi-relay cooperative wireless networks. IEEE Trans Wirel Commun 11(9):3250-3260

18. Phan KT, Nguyen DHN, Le-Ngoc T (2009) Joint power allocation and relay selection in cooperative networks, In: Proceedings of IEEE global telecommunications conference (GLOBECOM), Honolulu, Nov 30-Dec 4 2009, pp 1-5

19. Phan KT, Le-Ngoc T, Vorobyov SA (2009) Power allocation in wireless multi-user relay networks. IEEE Trans Wirel Commun 8(5):2535-2545

20. Rice JA (1995) Mathematical statistics and data analysis, 2nd edn. Duxbury Press, Belmont 